\title{
The association between new graphic health warning labels on tobacco products and attitudes toward smoking among south Korean adolescents: a national cross- sectional study
}

Ji-eun Hwang ${ }^{1}$ and Sung-il Cho ${ }^{1,2^{*}}$

\begin{abstract}
Background: Graphic health warning labels (GHWLs) on tobacco products are more effective than text warnings for communicating the risk of smoking. The implementation of GHWLs can prevent adolescents from initiating smoking. Therefore, this study examined the association between GHWLs newly implemented on December 23, 2016, in South Korea and attitudes toward smoking among adolescents.

Methods: This post-implementation cross-sectional analysis examined the responses of 62,276 students (31,624 boys and 30,652 girls) who participated in the 2017 Web-based Korean Youth Risk Behavior Survey, which was completed anonymously as a self-administered questionnaire by middle and high school students. Multinomial logistic regression was applied to explore the attitudes toward smoking among the youth (13-18 years old) who have been exposed to GHWLs in order to identify relationship of exposure to the GHWLs with smoking initiation and awareness of the danger of smoking.
\end{abstract}

Results: Six months after implementation, $69.4 \%$ of adolescents reported having been exposed to GHWLs in the previous 30 days. Among those exposed to GHWLs both boys and girls in grade 7 were significantly more likely than grade 12 high school students to decide not to start smoking (boys: $\mathrm{AOR}=3.96,95 \% \mathrm{Cl} 3.31-4.75, p<0.001$; girls: $\mathrm{AOR}=2.76,95 \% \mathrm{Cl} 2.32-3.30, p<0.001$ ) and to think that smoking was dangerous to their health (boys: $\mathrm{AOR}=3.01,95 \% \mathrm{Cl} 2.52-3.58, p<0.001$; girls: $\mathrm{AOR}=2.42,95 \% \mathrm{Cl} 2.03-3.88, p<0.001)$ after seeing GHWLs. These associations were greater for adolescents who had experienced smoking-prevention education or had been exposed to anti-tobacco advertisements. However, those who smoked, used e-cigarettes, or experienced secondhand smoking were significantly less likely to decide not to smoke and to view smoking as dangerous.

* Correspondence: persontime@hotmail.com

${ }^{1}$ Institute of Health and Environment, Seoul National University, 1 Gwanak-ro, Gwanak-gu, Seoul 08826, Republic of Korea

2Department of Public Health Science, Graduate School of Public Health, Seoul National University, 1 Gwanak-ro, Gwanak-gu, Seoul 08826, Republic of Korea

C The Author(s). 2020 Open Access This article is licensed under a Creative Commons Attribution 4.0 International License, which permits use, sharing, adaptation, distribution and reproduction in any medium or format, as long as you give appropriate credit to the original author(s) and the source, provide a link to the Creative Commons licence, and indicate if changes were made. The images or other third party material in this article are included in the article's Creative Commons licence, unless indicated otherwise in a credit line to the material. If material is not included in the article's Creative Commons licence and your intended use is not permitted by statutory regulation or exceeds the permitted use, you will need to obtain permission directly from the copyright holder. To view a copy of this licence, visit http://creativecommons.org/licenses/by/4.0/. The Creative Commons Public Domain Dedication waiver (http://creativecommons.org/publicdomain/zero/1.0/) applies to the data made available in this article, unless otherwise stated in a credit line to the data. 
(Continued from previous page)

Conclusions: To maintain the perception of the harm of tobacco from childhood through adolescence, the government should implement both comprehensive tobacco controls, including smoking-prevention education in schools, and measures to encourage a smoke-free environment in homes.

Keywords: Smoking, Tobacco, Graphic health warning labels, Adolescents, Education, Smoke-free

\section{Background}

Political and social changes that go beyond the regulation of tobacco products have been proposed for the tobacco endgame, which is a strategy to denormalize tobacco use and permanently remove tobacco products from society after a certain time point or to reduce smoking prevalence to a small percentage of the total population or in all population groups [1]. Although there is a lack of national consensus on a strategy [1], there is agreement about the necessity of ending the tobacco epidemic and protecting future generations from tobacco products $[2,3]$. To accomplish this goal, the nation must set a date for ending the tobacco epidemic and implement various measures that focus on the product, user, market, and supply chain. Efforts by both the government and the public to confront the tobacco industry are important [4].

However, because of the difficulties in achieving the tobacco endgame goal, the best initial approach would be to implement the Framework Convention on Tobacco Control (FCTC) fully. Whereas 181 countries have ratified the FCTC [5], others have opted not to participate. Also, the average implementation rate of the FCTC differs depending on which article in the treaty is being discussed [5]. Article 8 (protection from exposure to tobacco smoke) has been ratified by $88 \%$ of the participating countries compared to $17 \%$ for Article 17 (support for economically viable alternative activities) [5].

The measures in the FCTC are effective when they are carried out comprehensively [6, 7]. These measures interact to create a smoking cessation environment throughout society, increasing smoking cessation efforts and preventing initiation of smoking. The tobacco endgame is the key to creating a smoke-free generation [2, 3]; consequently, education, publicity, and policies for smoking prevention among children and adolescents are essential.

Many countries have implemented various tobacco control policies to prevent smoking among children and youth $[8,9]$. Increases in the price of tobacco products, school-based smoking-prevention education, smoke-free legislation, and mass media campaigns can prevent the initiation of smoking and reduce its prevalence among adolescents $[8,9]$.

Graphic health warning labels (GHWLs) on tobacco products are more effective than text warnings for communicating the risk of smoking [10]. Recently, regulations have been tightened, and standardized (plain), advertisement-free packaging has been implemented [11]. The implementation of such packaging may prevent the initiation of smoking by adolescents [10, 12]. However, only about half of FCTC countries have implemented GHWLs [5], and some have implemented one or two GHWLs on only $50 \%$ of tobacco products [5]. Such warnings are less effective than warnings on all tobacco products. Indeed, the warning pictures should be changed periodically to avoid familiarity, and their effectiveness should be assessed continuously [13].

GHWLs were implemented in South Korea on December 23, 2016 [14]. The 10 themes of the GHWLs are lung cancer, throat cancer, oral cancer, heart disease, stroke, secondhand smoke, smoking during pregnancy, impotence, skin aging, and premature death from cigarettes. Warnings are also printed on the packaging of electronic cigarettes (e-cigarettes), chewing tobacco, water pipes, and snus.

However, although exposure to the warning was examined just after the policy was initiated to assess its immediate impact, the South Korean policy has not been systematically investigated since then. Because one of the purposes of implementing GHWLs was to prevent the initiation of smoking by adolescents, it is important to evaluate whether adolescent awareness and attitudes have changed since the implementation. Studies in South Korea have found that text warnings on tobacco products are not effective in encouraging smoking cessation by current smokers $[15,16]$.

Therefore, this study investigated South Korean adolescents' exposure to, and the effect of, GHWLs. First, demographic data about the survey participants and South Korean adolescents in general were gathered. Then, participants' exposure to GHWLs during the previous 30 days, i.e., just after the policy was implemented, was determined, controlling for demographic characteristics associated with adolescents' smoking, and the perceived effectiveness of GHWLs was assessed, including participants' awareness of the harm of smoking and their intention to abstain from smoking after exposure to GHWLs. Sex differences in attitudes toward smoking were of particular interest [17-19]. Based on the results, a strategy for GHWLs and other tobacco-control policies targeted at adolescents are considered. 


\section{Methods}

\section{Participants}

Data for this study were obtained from the 2017 Korea

Youth Risk Behavior Web-based Survey (KYRBWS), which is conducted jointly by the Korean Ministry of Education, the Korean Ministry of Health and Welfare, and the Korean Center for Disease Control and Prevention to monitor the health behavior of South Korean adolescents [20]. The KYRBWS, a national cross-sectional survey [21], targeted middle and high school students in grades 7 to 12 in all regions of South Korea, including 5632 schools and 3,027,488 students [20]. To obtain a representative sample of students, the KYRBWS applied a complex sampling design including stratification, clustering, and multistage probability sampling [21]. All students in one class at 400 selected middle schools and 400 high schools participated in the survey [20]. In 2017, the survey was conducted from May to June, and the response rate was $95.8 \%$ [20]. In total, 62,276 students participated in the 2017 KYRBWS [20]; the present study analyzed data from all of them.

\section{Measurements}

The 2017 KYRBWS was conducted anonymously in a computer room, where each student completed a selfadministered, Web-based questionnaire consisting of 123 questions [20].

\section{Exposure to GHWLs during the past 30 days}

To assess their exposure to GHWLs, participants were asked the following: "For the past 30 days, did you see a graphic health warning on tobacco products?" The responses were classified as (1) No and (2) Yes.

\section{Perceived effectiveness of GHWLs after having viewed a warning}

This study used two questions from the Korea Youth Risk Behavior Web-based Survey. Participants who responded that they saw GHWLs in past 30 days were asked two questions: "When you saw the graphic health warning, did you think that you should not smoke?" and "When you saw the graphic health warning, did you think that smoking is harmful to health?". The responses to these questions were classified as (1) not at all, (2) slightly, (3) very, or (4) extremely. These questions are commonly used in the assessment of GHWLs and were taken from the peer-reviewed literature [22-25].

\section{Smoking status}

Cigarette-smoking status was assessed as the number of smoking days during the past 30 days. Respondents who reported smoking during $>1$ day were classified as current smokers, whereas those who reported smoking 0 days were classified as non-smokers. Respondents' e- cigarette smoking status was assessed by the number of days on which they used an e-cigarette during the past 30 days. Respondents who reported $>1$ days of e-cigarette use were classified as current users of e-cigarettes; respondents who reported 0 days of e-cigarette use were classified as non-users of e-cigarettes.

\section{Other indicators related to smoking}

Students were classified as exposed to secondhand smoke if someone in their household smoked during the previous 7 days. Students were considered to have experienced smoking-prevention education in school if they attended such a program during class time during the previous 12 months or if they were exposed to relevant educational broadcasts, participated in large group programs, and so on. Experience with anti-tobacco advertisements was defined as having seen or heard publicity about tobacco control during the previous 12 months (e.g., TV, radio, news, the Internet, newspapers, subways and bus stops, etc.). Exposure to tobacco advertisements was defined as having seen any tobacco advertisements in magazines, convenience stores, or supermarkets or on the Internet during the last 30 days.

\section{Covariates}

The analysis was controlled for the following demographic characteristics: gender, grade, educational stage, region, family living structure, perceived household economic status, perceived academic record, perceived stress, experience of depression, and current alcohol use. Regions were divided into metropolitan, medium and small cities, and rural. Family living structures were divided into three levels according to the current parental residence: living with both parents, living with a single parent, and other (living with grandparent, living with brother or sister, or living alone). Perceived household economic status was evaluated by the following question: "How would you rate your family's economic status?" The responses were re-categorized into three levels: wealthy (very wealthy or wealthy), medium income, and poor (poor or very poor). Perceived academic record was evaluated by the following question: "How would you describe your school grades?" Responses were recategorized in three levels: high (high and upper middle), middle, and low (lower middle and low).

Perceived stress was assessed by asking the following: "How much stress are you experiencing in your daily life?" The possible responses were (1) a very high level, (2) a high level, (3) a moderate level, (4) a low level, and (5) none. Experience of depression was assessed by asking the following: "In the past 12 months, have you experienced sadness or despair that interrupted your everyday life for a period of 2 weeks?" The responses were (1) Yes or (2) No. Current alcohol use was assessed 
as the number of drinking days during the past 30 days. Respondents who reported $>1$ days of drinking were classified as current alcohol users, whereas those reporting 0 days of drinking were classified as non-drinkers.

\section{Data analyses}

We conducted a statistical analysis of the KYRBWS that reflected the weights assigned considering the complex sampling survey design. A frequency analysis was conducted to assess the number and percentage of subjects according to demographic characteristics. The distribution of adolescents who had seen GHWLs in the past 30 days varied by gender and demographic characteristics. In addition, multinomial logistic regression was applied to explore the relationship between GHWLs and perceived effectiveness among students who had seen GHWLs on tobacco products in the past 30 days. In this test, the following covariates were adjusted for region, family living structure, perceived academic record, perceived household economic status, perceived stress, experience of depression, and current alcohol use; they are presented as adjusted odds ratios (AORs) and 95\% confidence intervals (CIs). All statistical analyses were stratified by gender. In addition, the data analyses were performed using SPSS ver. 24 , and $p$ values $<0.05$ were considered indicative of significance.

\section{Results}

\section{Demographic characteristics}

Approximately half $(51.4 \%$ in total; $51.3 \%$ of boys and $51.5 \%$ of girls) of the adolescents currently live in metropolitan areas, and the majority live with both parents (83.9\% in total; $83.5 \%$ of boys and $84.2 \%$ of girls) (Table 1 ). In addition, more than one-third evaluated their household economic level as medium (45.6\% in total; $42.9 \%$ of boys and $48.4 \%$ of girls) and their academic record as high (39.2\% in total; $40.8 \%$ of boys and $37.3 \%$ of girls). Most students reported stress in their lives (High $37.2 \%$ vs. Intermediate 42.6 vs. Low 20.2\%), and $25.1 \%$ of all students had experienced depression $(20.3 \%$ of boys and $30.3 \%$ of girls). Of the students, $16.1 \%$ (18.2\% of boys and $13.2 \%$ of girls) were current alcohol users.

\section{Exposure to GHWLs during the past 30 days}

More than two-thirds of the respondents $(69.4 \%$; $66.6 \%$ of boys and $72.4 \%$ of girls) reported that they had been exposed to GHWLs during the past 30 days. In boys, such exposure was most frequent among tenth graders; in girls, it was most frequent among ninth graders (Table 2). Current smokers ( $85.4 \%$ of boys and $87.7 \%$ of girls) and users of e-cigarettes (87.5\% of boys and $84.3 \%$ of girls) were more likely to be exposed to GHWLs than were non-smokers $(64.7 \%$ of boys and $71.9 \%$ of girls) and non-users $(65.9 \%$ of boys and $72.3 \%$ of girls).
Additionally, those who were exposed to secondhand smoke (boys: $75.6 \%$ vs. $63.3 \%$; girls: $80.7 \%$ vs. $69.0 \%$ ), tobacco advertisements (boys: $71.5 \%$ vs. $49.1 \%$; girls: $77.5 \%$ vs. $53.2 \%$ ), or anti-tobacco advertisements (boys: $71.8 \%$ vs. $47.1 \%$; girls: $75.6 \%$ vs. $51.8 \%$ ) were more likely to have been exposed to GHWLs than those who were not. In the past 12 months, $74.0 \%$ of boys and $76.5 \%$ of girls who had been educated about smoking prevention at school were exposed to GHWLs compared to only $50.6 \%$ of boys and $61.2 \%$ of girls who had not received such education.

Thinking about the harm of smoking after seeing a GHWL Table 3 shows the results of a multinomial logistic regression analysis (after adjustment for all covariates) of the relationship between perception of the harm of smoking and other variables among boys and girls who had seen GHWLs in the 30 days prior to the survey. The probability of thinking about smoking harm after exposure to GHWLs was higher among boys and girls in the lower grades than among those in the higher grades (seventh-grade boys: AOR 3.96, 95\% CI 3.314.75; seventh-grade girls: AOR 2.76, 95\% CI 2.32-0.30). The probability of thinking about the harm of smoking was significantly lower in smokers (boys: AOR 0.25, 95\% CI 0.23-0.28; girls: AOR 0.25, 95\% CI 0.21-0.30) than in non-smokers. Users of e-cigarettes (boys: AOR 0.85 , 95\% CI 0.72-0.99; girls: AOR 0.74, 95\% CI 0.531.05) were also less likely than non-users to think of the harm of smoking. Those who had received education regarding smoking prevention during school during the last 12 months were more likely to think about smoking-related harm compared to those who did not receive smoking-prevention education in school (boys: AOR 1.38, 95\% CI 1.26-1.50; girls: AOR 1.20, 95\% CI 1.10-1.32). Furthermore, the AOR for thinking about smoking harm was 1.48 (95\% CI 1.35-1.62) for boys and 1.85 (95\% CI 1.64-2.08) for girls who had been exposed to anti-tobacco advertisements during the past 12 months compared to those not exposed to such advertising.

\section{Intention not to start smoking after having seen GHWLs}

The results of the logistic regression analysis of the relationship between the intention not to start smoking after exposure to GHWLs during the previous 30 days and smoking-related variables, controlling for all covariates, are shown in Table 4. As with thinking about the harm of smoking, the intention not to start smoking was associated with grade, current smoking status, use of e-cigarettes, smoking-prevention education in school, and exposure to anti-tobacco advertisements between both genders. Also, boys who had been 
Table 1 Demographic characteristics of the survey participants $(n=62,276)$

\begin{tabular}{|c|c|c|c|c|c|c|}
\hline \multirow[t]{2}{*}{ Variable } & \multicolumn{2}{|c|}{ Total $(n=62,276)$} & \multicolumn{2}{|c|}{ Boys $(n=31,624)$} & \multicolumn{2}{|c|}{ Girls $(n=30,652)$} \\
\hline & $\bar{n}$ & $(\%)^{a}$ & $\bar{n}$ & $(\%)^{a}$ & $\bar{n}$ & $(\%)^{a}$ \\
\hline \multicolumn{7}{|l|}{ Grade } \\
\hline 7th grade & 10,189 & $(14.8)$ & 5178 & $(14.8)$ & 5011 & $(14.9)$ \\
\hline 8th grade & 10,377 & $(15.4)$ & 5272 & $(15.3)$ & 5105 & $(15.4)$ \\
\hline 9th grade & 10,319 & $(15.1)$ & 5202 & $(15.1)$ & 5117 & $(15.0)$ \\
\hline 10th grade & 10,165 & $(17.1)$ & 5069 & $(17.1)$ & 5096 & $(17.1)$ \\
\hline 11 th grade & 10,800 & (19.0) & 5610 & $(19.1)$ & 5190 & (18.9) \\
\hline 12th grade & 10,426 & (18.6) & 5293 & $(18.6)$ & 5133 & $(18.7)$ \\
\hline \multicolumn{7}{|l|}{ Educational stage } \\
\hline Middle school & 30,885 & $(45.3)$ & 15,652 & $(45.2)$ & 15,233 & $(45.3)$ \\
\hline High school & 31,391 & $(54.7)$ & 15,972 & $(54.8)$ & 15,419 & $(54.7)$ \\
\hline \multicolumn{7}{|l|}{ Region } \\
\hline Metropolitan & 32,065 & $(51.4)$ & 15,848 & $(51.3)$ & 16,217 & $(51.5)$ \\
\hline Medium and small cities & 26,614 & $(44.0)$ & 13,940 & $(44.2)$ & 12,674 & (43.9) \\
\hline Rural & 3597 & (4.6) & 1836 & $(4.5)$ & 1761 & (4.6) \\
\hline \multicolumn{7}{|l|}{ Family living structure } \\
\hline Living with both parents & 51,735 & (83.9) & 26,176 & $(83.5)$ & 25,559 & $(84.2)$ \\
\hline Living with a single parent & 8729 & $(13.5)$ & 4440 & $(13.5)$ & 4289 & $(13.4)$ \\
\hline Others & 1812 & (2.6) & 1008 & $(3.0)$ & 804 & (2.4) \\
\hline \multicolumn{7}{|c|}{ Perceived household economic status } \\
\hline Wealthy & 24,802 & $(40.4)$ & 13,618 & $(43.4)$ & 11,184 & $(37.1)$ \\
\hline Medium & 28,582 & (45.6) & 13,664 & $(42.9)$ & 14,918 & $(48.4)$ \\
\hline Poor & 8892 & $(14.0)$ & 4342 & $(13.7)$ & 4550 & $(14.5)$ \\
\hline \multicolumn{7}{|l|}{ Perceived academic record } \\
\hline High & 24,524 & $(39.2)$ & 12,925 & $(40.8)$ & 11,599 & $(37.3)$ \\
\hline Middle & 17,810 & (28.7) & 8683 & $(27.6)$ & 9127 & $(30.0)$ \\
\hline Low & 19,942 & (32.1) & 10,016 & (31.6) & 9926 & (32.7) \\
\hline \multicolumn{7}{|l|}{ Perceived stress } \\
\hline High & 23,259 & $(37.2)$ & 9552 & $(30.4)$ & 13,707 & $(44.6)$ \\
\hline Middle & 26,271 & $(42.6)$ & 13,745 & $(43.9)$ & 12,526 & $(41.2)$ \\
\hline Low & 12,746 & (20.2) & 8327 & $(25.8)$ & 4419 & $(14.2)$ \\
\hline \multicolumn{7}{|l|}{ Depression experience } \\
\hline Yes & 15,612 & (25.1) & 6326 & $(20.3)$ & 9286 & (30.3) \\
\hline No & 46,664 & $(74.9)$ & 25,298 & $(79.7)$ & 21,366 & $(69.7)$ \\
\hline \multicolumn{7}{|l|}{ Current alcohol use } \\
\hline User & 9597 & $(16.1)$ & 5562 & $(18.2)$ & 4035 & (13.2) \\
\hline Non-user & 52,679 & (83.9) & 26,062 & (81.8) & 26,617 & (86.8) \\
\hline
\end{tabular}

${ }^{a}$ Weighted percentages considering the complex sample survey design

exposed to secondhand smoke during the past 7 days were significantly less likely to report the intention to start smoking than were boys (boys: AOR 0.85, 95\% CI 0.79-0.92; girls: AOR 0.96, 95\% CI 0.88-1.05) not exposed to secondhand smoke. The AOR was 1.03 (95\% CI 0.921.15) for boys and 1.22 (95\% CI 1.10-1.35) for girls who had been exposed to tobacco advertisements during past 30 days, but this was significant only in girls.

\section{Discussion}

We examined whether adolescents had been exposed to GHWLs and whether such exposure was associated with 
Table 2 Prevalence of exposure to graphic health warnings on tobacco products for the past 30 days among South Korean adolescents $(n=62,276)$

\begin{tabular}{|c|c|c|c|c|c|c|c|c|}
\hline \multirow[t]{3}{*}{ Variable } & \multicolumn{4}{|c|}{ Boys $(n=31,624)$} & \multicolumn{4}{|c|}{ Girls $(n=30,652)$} \\
\hline & \multicolumn{2}{|l|}{ Yes } & \multicolumn{2}{|l|}{ No } & \multicolumn{2}{|l|}{ Yes } & \multicolumn{2}{|l|}{ No } \\
\hline & $\bar{n}$ & $(\%)^{a}$ & $\bar{n}$ & $(\%)^{a}$ & $\bar{n}$ & $(\%)^{a}$ & $\bar{n}$ & $(\%)^{a}$ \\
\hline \multicolumn{9}{|l|}{ Grade } \\
\hline 7th grade & 3425 & $(66.9)$ & 1753 & $(33.1)$ & 3599 & (72.3) & 1412 & $(27.7)$ \\
\hline 8th grade & 3467 & $(66.0)$ & 1805 & $(34.0)$ & 3711 & (72.9) & 1394 & $(27.1)$ \\
\hline 9th grade & 3401 & $(65.5)$ & 1801 & $(34.5)$ & 3749 & (73.0) & 1368 & $(27.0)$ \\
\hline 10th grade & 3482 & $(68.6)$ & 1587 & $(31.4)$ & 3654 & (71.6) & 1442 & $(28.4)$ \\
\hline 11th grade & 3703 & $(65.8)$ & 1907 & $(34.2)$ & 3746 & (72.2) & 1444 & $(27.8)$ \\
\hline 12th grade & 3576 & $(67.0)$ & 1717 & $(33.0)$ & 3718 & (72.3) & 1415 & $(27.7)$ \\
\hline \multicolumn{9}{|c|}{ Current smoking status } \\
\hline Smoker & 2437 & $(85.4)$ & 421 & $(14.6)$ & 789 & $(87.7)$ & 122 & (12.3) \\
\hline Non-Smoker & 18,617 & $(64.7)$ & 10,149 & $(35.3)$ & 21,388 & (71.9) & 8353 & $(28.1)$ \\
\hline \multicolumn{9}{|l|}{ e-cigarette status } \\
\hline User & 864 & $(87.5)$ & 127 & $(12.5)$ & 214 & (85.3) & 39 & $(14.7)$ \\
\hline Non-User & 20,190 & $(65.9)$ & 10,443 & $(34.1)$ & 21,963 & (72.3) & 8436 & $(27.7)$ \\
\hline \multicolumn{9}{|c|}{ Secondhand Smoke } \\
\hline Non-exposed & 14,407 & $(63.3)$ & 8383 & $(36.7)$ & 14,978 & $(69.0)$ & 6743 & $(31.0)$ \\
\hline Exposed & 6647 & $(75.6)$ & 2187 & $(24.4)$ & 7199 & $(80.7)$ & 1732 & (19.3) \\
\hline \multicolumn{9}{|c|}{ Tobacco Advertise } \\
\hline Non-exposed & 3361 & $(49.1)$ & 3505 & $(50.9)$ & 3408 & $(53.2)$ & 2978 & $(46.8)$ \\
\hline Exposed & 17,693 & $(71.5)$ & 7065 & $(28.5)$ & 18,769 & (77.5) & 5497 & $(22.5)$ \\
\hline \multicolumn{9}{|c|}{ Anti-Tobacco Advertisement } \\
\hline Non-exposed & 3055 & $(47.1)$ & 3455 & $(52.9)$ & 2141 & (51.8) & 1984 & $(48.2)$ \\
\hline Exposed & 17,999 & $(71.8)$ & 7115 & $(28.2)$ & 20,036 & (75.6) & 6491 & $(24.4)$ \\
\hline \multicolumn{9}{|c|}{ Experience of smoking prevention education in school } \\
\hline Yes & 16,218 & $(74.0)$ & 5806 & $(26.0)$ & 17,379 & $(76.5)$ & 5395 & $(23.5)$ \\
\hline No & 4836 & $(50.6)$ & 4764 & $(49.4)$ & 4798 & $(61.2)$ & 3080 & (38.8) \\
\hline
\end{tabular}

${ }^{a}$ Weighted percentages considering the complex sample survey design

their perception of smoking. The results show that most youth had been exposed to GHWLs during the previous month and that such exposure had enhanced their intention not to start smoking. The GHWLs also effectively communicated the risks of smoking. Exposure to GHWLs was most strongly associated with prior experience with other smoking-prevention education or antitobacco advertisements. Furthermore, current smokers, current users of e-cigarettes, and those exposed to secondhand smoke were less associated by the GHWLs than were those who had not had such experiences.

As young people progress in school, the probability that they will consider smoking to be harmful to health is reduced, as is the likelihood that they will not consider starting to smoke. Just as the increase in age or grade is strongly associated with smoking initiation in adolescents [26], risky behaviors, such as drinking alcohol and smoking, also peak as a function of aging [27]. Because youth are prone to engage in risky behavior during adolescence, it will be difficult to prevent smoking in this population using GHWLs alone [28]. To prevent smoking, it is important that a well-formed negative attitude toward smoking continue from childhood into adolescence so that this same attitude can be carried into adulthood [29, 30]. For this reason, young people should be continually educated, starting in childhood, about the harm of smoking and the benefits of not smoking; such efforts will eliminate curiosity about smoking and help youth resist the temptation to start smoking.

However, young people who already smoked were less associated by the content of the GHWLs, and they were less likely to see smoking as harmful than were nonsmokers who saw the warnings. This may be due to cognitive dissonance and defensiveness among those who already smoked [31-34]. Changing the attitudes and awareness of young smokers requires a more persuasive 
Table 3 The relationship between perception about the harm of smoking and other variables after exposure to graphic health warnings during the previous 30 days by South Korean boys and girls

\begin{tabular}{|c|c|c|c|c|c|c|c|c|}
\hline \multirow[t]{2}{*}{ Variable } & \multicolumn{4}{|l|}{ Boys } & \multicolumn{4}{|l|}{ Girls } \\
\hline & $\bar{N}$ & $(\%)^{a}$ & $\mathrm{AOR}^{\mathrm{b}}$ & $(95 \% \mathrm{Cl})$ & $\bar{n}$ & $(\%)^{a}$ & $\mathrm{AOR}^{\mathrm{b}}$ & $(95 \% \mathrm{Cl})$ \\
\hline \multicolumn{9}{|l|}{ Grade } \\
\hline 7th grade & 3242 & $(94.6)$ & 3.96 & $(3.31-4.75)$ & 3390 & $(94.0)$ & 2.76 & $(2.32-3.30)$ \\
\hline 8th grade & 3082 & $(88.3)$ & 1.93 & $(1.67-2.23)$ & 3356 & $(90.0)$ & 1.77 & $(1.52-2.06)$ \\
\hline 9th grade & 2899 & $(85.0)$ & 1.63 & $(1.41-1.88)$ & 3337 & $(89.0)$ & 1.65 & $(1.43-1.90)$ \\
\hline 10th grade & 2726 & $(78.0)$ & 1.16 & $(1.02-1.31)$ & 3165 & $(86.4)$ & 1.42 & $(1.25-1.60)$ \\
\hline 11th grade & 2773 & $(74.2)$ & 1.10 & $(0.98-1.23)$ & 3110 & $(83.1)$ & 1.16 & $(1.02-1.31)$ \\
\hline 12th grade & 2546 & $(70.6)$ & 1.00 & & 2990 & $(80.2)$ & 1.00 & \\
\hline \multicolumn{9}{|c|}{ Current smoking status } \\
\hline Smoker & 1180 & $(47.7)$ & 0.25 & $(0.23-0.28)$ & 404 & $(52.4)$ & 0.25 & $(0.21-0.30)$ \\
\hline Non-Smoker & 16,088 & $(85.6)$ & 1.00 & & 18,944 & $(88.1)$ & 1.00 & \\
\hline \multicolumn{9}{|l|}{ e-cigarette status } \\
\hline User & 427 & $(49.7)$ & 0.85 & $(0.72-0.99)$ & 108 & $(52.1)$ & 0.74 & $(0.53-1.05)$ \\
\hline Non-User & 16,841 & $(82.4)$ & 1.00 & & 19,240 & $(87.1)$ & 1.00 & \\
\hline \multicolumn{9}{|c|}{ Expose to Secondhand Smoke } \\
\hline Exposed & 5317 & $(78.9)$ & 0.97 & $(0.90-1.05)$ & 6174 & $(85.4)$ & 0.99 & $(0.90-1.09)$ \\
\hline Non-exposed & 11,951 & $(81.9)$ & 1.00 & & 13,174 & $(87.4)$ & 1.00 & \\
\hline \multicolumn{9}{|c|}{ Tobacco Advertise } \\
\hline Exposed & 14,627 & $(81.6)$ & 1.09 & $(0.98-1.22)$ & 16,453 & $(87.3)$ & 1.11 & $(0.99-1.24)$ \\
\hline Non-exposed & 2641 & $(77.6)$ & 1.00 & & 2895 & $(83.8)$ & 1.00 & \\
\hline \multicolumn{9}{|c|}{ Anti-Tobacco Advertisement } \\
\hline Exposed & 15,057 & $(82.7)$ & 1.48 & $(1.35-1.62)$ & 17,699 & $(87.9)$ & 1.85 & $(1.64-2.08)$ \\
\hline Non-exposed & 2211 & $(71.2)$ & 1.00 & & 1649 & $(76.3)$ & 1.00 & \\
\hline \multicolumn{9}{|c|}{ Experience of smoking prevention education in school } \\
\hline Yes & 13,580 & $(82.9)$ & 1.38 & $(1.26-1.50)$ & 15,325 & (87.8) & 1.20 & $(1.10-1.32)$ \\
\hline No & 3688 & $(74.9)$ & 1.00 & & 4023 & (83.3) & 1.00 & \\
\hline
\end{tabular}

AOR Adjusted odds ratio, $\mathrm{Cl}$ Confidence interval

${ }^{a}$ Weighted percentages considering the complex sample survey design

bobtained from multinomial logistic regression analysis with control variables; Region, Family living structure, Perceived academic record, Perceived household economic status, Perceived stress, Depression experience, Current alcohol use

warning. Adolescent smokers prefer loss- rather than gain-framed warnings [35], and the guilt and fear arising from such warnings tend to reinforce their impact [36]. Additionally, an environment in which curiosity-inducing factors are blocked beforehand is required, as is a continued understanding of why adolescents smoke.

Smoking susceptibility is an important predictor of smoking in adolescents. The susceptibility to smoking is related to several variables, including genetic (e.g., race, ethnicity) [37], environmental (e.g., exposure to tobacco advertisements) [38], and psychosocial and lifestyle (e.g., aggression, depression, anxiety, sensation-seeking) factors $[39,40]$. In particular, exposure to secondhand smoke not only adversely affects the health of infants and young children [41] but also increases their likelihood of smoking [42]. Indeed, secondhand smoke exposure is associated with a greater likelihood of being a smoker and of initiating smoking [42].

Although significant only in boys, exposure to secondhand smoke during the past 7 days was associated with a reduced intention not to start smoking in this study. Continuous exposure to secondhand smoke may lead to underestimation of the risk of using tobacco products and to familiarity with them. As a result, GHWLs alone are insufficient to change the perceptions and attitudes of tobacco-friendly adolescents [43], such as current smokers who are familiar with tobacco products and those exposed to secondhand smoke. These results suggest that a single policy is inadequate for the prevention of smoking by adolescents and underscore the need for multiple integrated approaches.

Smoking-prevention education for students is not limited to simply informing them about the risk of smoking; 
Table 4 The relationship between the intention not to start smoking and other variables after exposure to graphic health warnings during the past 30 days among South Korean boys and girls

\begin{tabular}{|c|c|c|c|c|c|c|c|c|}
\hline \multirow[t]{2}{*}{ Variable } & \multicolumn{4}{|l|}{ Boys } & \multicolumn{4}{|l|}{ Girls } \\
\hline & $\mathrm{N}$ & $(\%)^{a}$ & $\mathrm{AOR}^{\mathrm{b}}$ & $(95 \% \mathrm{Cl})$ & $n$ & $(\%)^{a}$ & $\mathrm{AOR}^{\mathrm{b}}$ & $(95 \% \mathrm{Cl})$ \\
\hline \multicolumn{9}{|l|}{ Grade } \\
\hline 7th grade & 3190 & $(93.2)$ & 3.01 & $(2.52-3.58)$ & 3393 & (94.3) & 2.42 & $(2.03-2.88)$ \\
\hline 8th grade & 3041 & $(87.1)$ & 1.78 & $(1.56-2.04)$ & 3358 & $(90.1)$ & 1.58 & $(1.36-1.84)$ \\
\hline 9th grade & 2850 & $(83.6)$ & 1.58 & $(1.37-1.82)$ & 3334 & $(88.8)$ & 1.49 & $(1.29-1.73)$ \\
\hline 10th grade & 2693 & $(77.2)$ & 1.23 & $(1.09-1.40)$ & 3180 & $(87.0)$ & 1.43 & $(1.25-1.63)$ \\
\hline 11th grade & 2676 & $(71.5)$ & 1.11 & $(1.00-1.24)$ & 3147 & $(84.1)$ & 1.26 & $(1.11-1.43)$ \\
\hline 12th grade & 2431 & $(67.3)$ & 1.00 & & 2985 & $(80.4)$ & 1.00 & \\
\hline \multicolumn{9}{|c|}{ Current smoking status } \\
\hline Smoker & 817 & $(32.1)$ & 0.15 & $(0.13-0.16)$ & 264 & (33.6) & 0.13 & $(0.11-0.16)$ \\
\hline Non-Smoker & 16,064 & $(85.6)$ & 1.00 & & 19,133 & $(89.1)$ & 1.00 & \\
\hline \multicolumn{9}{|l|}{ e-cigarette status } \\
\hline User & 285 & $(32.5)$ & 0.64 & $(0.53-0.78)$ & 63 & $(29.4)$ & 0.50 & $(0.32-0.78)$ \\
\hline Non-User & 16,596 & $(81.2)$ & 1.00 & & 19,334 & $(87.7)$ & 1.00 & \\
\hline \multicolumn{9}{|c|}{ Expose to Secondhand Smoke } \\
\hline Exposed & 5073 & $(74.8)$ & 0.85 & $(0.79-0.92)$ & 6127 & $(84.7)$ & 0.96 & $(0.88-1.05)$ \\
\hline Non-exposed & 11,808 & $(81.1)$ & 1.00 & & 13,270 & $(88.2)$ & 1.00 & \\
\hline \multicolumn{9}{|l|}{ Tobacco Advertise } \\
\hline Exposed & 14,272 & $(79.5)$ & 1.03 & $(0.92-1.15)$ & 16,507 & (87.6) & 1.22 & $(1.10-1.35)$ \\
\hline Non-exposed & 2609 & $(76.9)$ & 1.00 & & 2890 & (83.9) & 1.00 & \\
\hline \multicolumn{9}{|c|}{ Anti-Tobacco Advertisement } \\
\hline Exposed & 14,701 & $(80.7)$ & 1.34 & $(1.23-1.47)$ & 17,710 & $(88.0)$ & 1.55 & $(1.35-1.77)$ \\
\hline Non-exposed & 2180 & $(70.2)$ & 1.00 & & 1687 & $(78.4)$ & 1.00 & \\
\hline \multicolumn{9}{|c|}{ Experience of smoking prevention education in school } \\
\hline Yes & 13,267 & $(81.0)$ & 1.40 & $(1.28-1.53)$ & 15,361 & $(88.0)$ & 1.23 & $(1.12-1.36)$ \\
\hline No & 3614 & $(73.2)$ & 1.00 & & 4036 & $(83.8)$ & 1.00 & \\
\hline
\end{tabular}

AOR Adjusted odds ratio, $\mathrm{Cl}$ Confidence interval

a'Weighted percentages considering the complex sample survey design

${ }^{b}$ Obtained from multinomial logistic regression analysis with control variables; Region, Family living structure, Perceived academic record, Perceived household economic status, Perceived stress, Depression experience, Current alcohol use

it can also help to strengthen the negative opinions about smoking, thus preventing smoking initiation [44]. In this study, educated adolescents were more aware of the health consequences of the warning picture than were those who were not educated, and the hesitancy of the former group to start smoking was strengthened. Thus, smoking-prevention education at school is important for tobacco control because it positively affects the impact of relevant policies $[45,46]$. To maximize the effectiveness of smoking-prevention education, it should be coordinated with other tobacco-control policies to prevent smoking among adolescents $[45,46]$.

Anti-tobacco advertisements are also part of tobaccocontrol policy and are aimed at preventing smoking initiation in youth. However, among teenagers, GHWLs led to a greater health awareness and stronger non-smoking intent than did anti-tobacco advertisements alone.
Because promotion of smoking cessation or prevention of smoking is more effective when used in combination with other methods, multiple approaches should be implemented [47]. Therefore, tobacco-control policies and messages must be promoted to increase awareness of the effects of tobacco products and smoking and to promote negative attitudes toward them.

Our results have important implications for the tobacco endgame. The tobacco-free-generation approach, a key endgame strategy, is based on the goal of legally preventing individuals born after a certain year from accessing tobacco products [2, 3]. Public support for such a mandatory approach would be enhanced by tobacco denormalization, and plain packaging is a useful step toward this goal. Because denormalization strategies typically focus on tobacco use or tobacco users [2, 3], a plain-packaging policy has greater potential as it serves 
to denormalize tobacco products, thus justifying subsequent legal measures. However, our results suggest that denormalization of tobacco products may have limited efficacy in youth who have experienced active or passive smoking. Indeed, a focus on a particular cohort may not be effective, as extant attitudes and experiences may blunt the impact of such an approach. The logical follow up to tobacco product denormalization by means of packaging policy is denormalization of the tobacco industry, which justifies control of the supply chain. We suggest that future anti-smoking policies focus on denormalization of the tobacco industry.

Tobacco-packaging policy, whether strong or weak, removes the attraction of tobacco and reduces its value so that it is not worth purchasing. Currently, packaging policy concentrates on denormalizing the tobacco product by imparting information about its harmful effects. It is important to continue strengthening packaging policy so that the packaging of all tobacco products is plain. However, any packaging policy is only one of the various comprehensive measures required to denormalize the tobacco industry, to eradicate the primary force driving smoking [48]. Research on how the packaging contents impart this message, and the combinations of policies required to achieve denormalization of the tobacco industry, is needed.

This study has several limitations. First, the survey was performed from May to June 2017, a period that differed from that of the exposure to the GHWLs. Second, the exposure pathway of the GHWLs could not be determined precisely; possibilities include the media, points of sale, school curriculum, and/or cigarette packs. These different pathways of exposure may have affected the perceptions of and attitudes toward smoking and should be evaluated as part of future studies. Third, the students who had seen GHWLs were asked about their future intention not to start smoking, but this study did not compare their responses to those of youth who had not seen GHWLs; this should be the subject of future work. Finally, because this was a cross-sectional study, we examined the relationship between the implementation of new GHWLs and their perceived effectiveness on adolescents' attitudes toward smoking; we could not assess the causal relationship between GHWLs and smoking attitudes. Also, since this study used secondary data, the researchers cannot be involved in selecting research instruments to measure the effect of GHWLs exposure. For long-term analysis, various instruments with validity and accuracy are needed for multilateral assessment of the effect of exposure to GHWLs. It is necessary to establish a long-term cohort study to examine whether GHWLs are effective in deterring adolescents from initiating smoking and, if so, what the degree of that effectiveness is.

\section{Conclusion}

The GHWLs strengthened the perception of the risk of smoking and the intention not to start smoking among adolescents. The positive association of GHWLs with risk awareness was greater in adolescents who had experienced smoking-prevention education or who had been exposed to anti-tobacco advertisements. By contrast, the association decreased among those who were already smokers or users of e-cigarettes, as well as in students in higher grades and young people exposed to secondhand smoke at home. Comprehensive school-based smoking-prevention education and efforts to promote smoke-free home environments are needed to help adolescents carry their negative perceptions about smoking into adulthood.

\section{Abbreviations}

FCTC: Framework Convention on Tobacco Control; GHWLs: Graphic Health Warning Labels; KYRBWS: Korea Youth Risk Behavior Web-based Survey; AORs: adjusted odds ratios; Cls: confidence intervals

\section{Acknowledgements}

The authors thank the Korean Center for disease Control and Prevention $(\mathrm{KCDC})$.

\section{Authors' contributions}

$J \mathrm{H}$ designed the study, analyzed and interpreted the data, and wrote the manuscript. SC contributed to the study design and interpretation of the results. The authors read and approved the final manuscript.

\section{Funding}

This work was supported by the National Research Foundation of Korea(NRF) grant funded by the Korea government(MSIT) (No. 2020R1C1C1012562).

\section{Availability of data and materials}

The microdata of KYRBS are released annually in December each year [21]. The datasets analyzed during the current study are available on the KYRBS website, http://yhs.cdc.go.kr.

\section{Ethics approval and consent to participate}

A secondary analysis of existing data with human subjects may be exempted from examination by the competent institutional committee according to Korean law (Bioethics and Safety Act). Therefore, this study was approved for approved for exemption consent by the Institutional Review Board of the Seoul National University (No. E1811/002-002).

Consent for publication

Not Applicable.

\section{Competing interests}

The authors declare that they have no competing interests.

Received: 23 March 2019 Accepted: 2 April 2020

Published online: 24 May 2020

\section{References}

1. McDaniel PA, Smith EA, Malone RE. The tobacco endgame: a qualitative review and synthesis. Tob Control. 2016;25(5):594-604.

2. Khoo D, Chiam Y, Ng P, Berrick AJ, Koong HN. Phasing-out tobacco: proposal to deny access to tobacco for those born from 2000. Tob Control. 2010;19(5):355-60.

3. Berrick AJ. The tobacco-free generation proposal. Tob Control. 2013; 22(Suppl 1):i22-6.

4. Wilson N, Hoek J, Thomson G, Blakely T, Edwards R. Fifty years since the Royal College Report: more action needed to achieve the "Smokefree New Zealand by 2025" goal. N Z Med J. 2012;125(1351):109-12. 
5. World Health Organization. 2018 GLOBAL PROGRESS REPORT on implementation of the WHO framework convention on tobacco control; 2018.

6. Wakefield M, Chaloupka F. Effectiveness of comprehensive tobacco contro programmes in reducing teenage smoking in the USA. Tob Control. 2000; 9(2):177-86.

7. Stephens T, Pederson LL, Koval JJ, Macnab J. Comprehensive tobacco control policies and the smoking behaviour of Canadian adults. Tob Control. 2001;10(4):317-22.

8. Crawford MA, Balch Gl, Mermelstein R. Responses to tobacco control policies among youth. Tob Control. 2002;11(1):14-9.

9. Hawkins SS, Bach N, Baum CF. Impact of tobacco control policies on adolescent smoking. J Adolesc Health. 2016;58(6):679-85.

10. Hammond D. Health warning messages on tobacco products: a review. Tob Control. 2011:20(5):327-37.

11. Moodie C, Hoek J, Scheffels J, Gallopel-Morvan K, Lindorff K. Plain packaging: legislative differences in Australia, France, the UK, New Zealand and Norway, and options for strengthening regulations. Tob Control. 2018; 28(5):485.

12. Noar SM, Hall MG, Francis DB, Ribisl KM, Pepper JK, Brewer NT. Pictorial cigarette pack warnings: a meta-analysis of experimental studies. Tob Control. 2016;25(3):341-54

13. World Health Organization. Guidelines for implementation of article 11: packaging and labelling of tobacco products; 2008.

14. Kang E. Assessing health impacts of pictorial health warning labels on cigarette packs in Korea using DYNAMO-HIA. J Prev Med Public Health. 2017;50(4):251-61.

15. Kim HS, Bae HK, Suh YS, Kim DH, Shon KS, Bae IH. An evaluation on the health warning messages and graphic on cigarette packages in smoking students. J Korean Acad Fam Med. 2004:25(6):469-74.

16. Cho KS, Shin YJ. A survey on the effect of cigarette warning labels. J Korean Acad Fam Med. 2006;27(2):128-35.

17. Urberg K, Robbins RL. Adolescents' perceptions of the costs and benefits associated with cigarette smoking: sex differences and peer influence. J Youth Adolesc. 1981;10(5):353-61.

18. Carter OBJ, Donovan RJ, Weller NM, Jalleh G. Impact of smoking images in magazines on the smoking attitudes and intentions of youth: an experimental investigation. Tob Control. 2007:16(6):368-72.

19. Waldron I. Patterns and causes of gender differences in smoking. Soc Sci Med. 1991:32(9):989-1005.

20. Korean Ministry of Education, Korean Ministry of Health and Welfare. Prevention KCfdCa, Xiaoli N: 2017 Korean youth risk behavior web-based survey; 2017.

21. Kim Y, Choi S, Chun C, Park S, Khang YH, Oh K. Data resource profile: the Korea youth risk behavior web-based survey (KYRBS). Int J Epidemiol. 2016; 45(4): 1076.

22. Chang FC, Chung CH, Yu PT, Chao KY. The impact of graphic cigarette warning labels and smoke-free law on health awareness and thoughts of quitting in Taiwan. Health Educ Res. 2011;26(2):179-91.

23. Hammond D, Fong GT, McDonald PW, Cameron R, Brown KS. Impact of the graphic Canadian warning labels on adult smoking behaviour. Tob Control. 2003;12(4):391-5

24. Moodie C, Mackintosh AM, Hastings G. Adolescents' response to pictoria warnings on the reverse panel of cigarette packs: a repeat cross-sectional study. Tob Control. 2015;24(e1):e93-7.

25. Gravely S, Fong GT, Driezen P, McNally M, Thrasher JF, Thompson ME, et al. The impact of the 2009/2010 enhancement of cigarette health warning labels in Uruguay: longitudinal findings from the international tobacco control (ITC) Uruguay survey. Tob Control. 2016;25(1):89-95

26. O'Loughlin J, Paradis G, Renaud L, Gomez LS. One-year predictors of smoking initiation and of continued smoking among elementary schoolchildren in multiethnic, low-income, inner-city neighbourhoods. Tob Control. 1998;7(3):268-75

27. Duell N, Steinberg L, Icenogle G, Chein J, Chaudhary N, Di Giunta L, et al. Age patterns in risk taking across the world. J Youth Adolesc. 2018;47(5): 1052-72.

28. Steinberg L. Risk taking in adolescence: new perspectives from brain and behavioral science. Curr Dir Psychol Sci. 2007;16(2):55-9.

29. HarrelL JS, Bangdiwala SI, Deng S, Webb JP, Bradley C. Smoking initiation in youth: the roles of gender, race, socioeconomics, and developmental status. J Adolesc Health. 1998;23(5):271-9.
30. Wiehe SE, Garrison MM, Christakis DA, Ebel BE, Rivara FP. A systematic review of school-based smoking prevention trials with long-term follow-up. J Adolesc Health. 2005;36(3):162-9.

31. Stewart DW, Martin IM. Intended and unintended consequences of warning messages: a review and synthesis of empirical research. J Public Policy Mark. 1994;13(1):1-19.

32. Erceg-Hurn DM, Steed LG. Does exposure to cigarette health warnings elicit psychological reactance in smokers? J Appl Soc Psychol. 2011;41(1):219-37.

33. Johnson SE, Wu CC, Coleman BN, Choiniere CJ. Self-reported exposure to tobacco warning labels among U.S. middle and high school students. Am J Prev Med. 2014:47(2 Suppl 1):S69-75.

34. Andrews JC, Netemeyer RG, Kees J, Burton S. How graphic visual health warnings affect young smokers' thoughts of quitting. J Mar Res. 2014;51(2): $165-83$

35. Goodall C, Appiah O. Adolescents' perceptions of Canadian cigarette package warning labels: investigating the effects of message framing. Health Commun. 2008;23(2):117-27.

36. Netemeyer RG, Burton S, Andrews JC, Kees J. Graphic health warnings on cigarette packages: the role of emotions in affecting adolescent smoking consideration and secondhand smoke beliefs. J Public Policy Mark. 2016; 35(1):124-43.

37. El-Toukhy S, Sabado M, Choi K. Trends in susceptibility to smoking by race and ethnicity. Pediatrics. 2016;138:5.

38. Feighery EC, Henriksen L, Wang Y, Schleicher NC, Fortmann SP. An evaluation of four measures of adolescents' exposure to cigarette marketing in stores. Nicotine Tob Res. 2006;8(6):751-9.

39. Colder CR, Scalco M, Trucco EM, Read JP, Lengua LJ, Wieczorek WF, et al. Prospective associations of internalizing and externalizing problems and their co-occurrence with early adolescent substance use. J Abnorm Child Psychol. 2013;41(4):667-77.

40. Leeman RF, Hoff RA, Krishnan-Sarin S, Patock-Peckham JA, Potenza MN. Impulsivity, sensation-seeking, and part-time job status in relation to substance use and gambling in adolescents. J Adolesc Health. 2014;54(4): 460-6.

41. U.S. Department of Health and Human Services. Preventing tobacco use among youth and young adults: a report of the surgeon general. Atlanta: U. S. Department of Health and Human Services, Centers for Disease Control and Prevention, National Center for Chronic Disease Prevention and Health Promotion, Office on Smoking and Health; 2012.

42. Okoli CT, Kodet J. A systematic review of secondhand tobacco smoke exposure and smoking behaviors: smoking status, susceptibility, initiation, dependence, and cessation. Addict Behav. 2015:47:22-32.

43. Van Dessel P, Smith CT, De Houwer J. Graphic cigarette pack warnings do not produce more negative implicit evaluations of smoking compared to text-only warnings. PLoS One. 2018;13(3):e0194627.

44. Dobbins M, DeCorby K, Manske S, Goldblatt E. Effective practices for schoolbased tobacco use prevention. Prev Med. 2008;46(4):289-97.

45. Backinger $\mathrm{CL}$, Fagan P, Matthews E, Grana R. Adolescent and young adult tobacco prevention and cessation: current status and future directions. Tob Control. 2003:12(suppl 4):iv46-53.

46. Levy DT, Chaloupka F, Gitchell J. The effects of tobacco control policies on smoking rates: a tobacco control scorecard. J Public Health Manag Pract. 2004;10(4):338-53.

47. Thrasher JF, Murukutla N, Perez-Hernandez R, Alday J, Arillo-Santillan E, Cedillo C, et al. Linking mass media campaigns to pictorial warning labels on cigarette packages: a cross-sectional study to evaluate effects among Mexican smokers. Tob Control. 2013:22(e1):e57-65.

48. Proctor RN. Why ban the sale of cigarettes? The case for abolition. Tob Control. 2013:22(Suppl 1):i27-30.

\section{Publisher's Note}

Springer Nature remains neutral with regard to jurisdictional claims in published maps and institutional affiliations. 